10

\title{
Spectroscopic Analysis of Fluorescent Proteins Infiltrated into Photonic
} Crystals*

\author{
(C) N. Zhdanova ${ }^{1}$, A. Pakhomov ${ }^{2}$, S. Rodionov ${ }^{3}$, Yu. Strokova $^{1}$, S. Svyakhovskiy ${ }^{1}$, and A. Saletskii ${ }^{1}$ \\ ${ }^{1}$ Lomonosov Moscow State university, \\ 119991 Moscow, Russia \\ ${ }^{2}$ Shemyakin-Ovchinnikov Institute of Bioorganic Chemistry, Russian Academy of Sciences, \\ 117997 Moscow, Russia \\ ${ }^{2}$ N.N. Priorov Central Institute for Traumatology and Orthopedics, \\ 127299 Moscow, Russia \\ e-mail: zhdanova@physics.msu.ru
}

Received December 16, 2019

Revised January 07, 2020

Accepted February 28, 2020

Spectral properties of enhanced-green uorescent protein and monomeric red uorescent protein in porous photonic structures have been studied. The uorescent proteins were successfully inЛtrated into porous silicon photonic structures with dirent positions of the photonic band gap in visible spectral range. The intensity of uorescence is enhanced in the spectral regions of high photonic density of states. The possibility to control the uorescence spectra by the structure with the photonic band gap is demonstrated.

Keywords: photonic crystals, porous silicon, uorescent proteins, photonic band gap.

* Полный текст статьи опубликован в „Optics and Spectroscopy“ V. 128 N 72020. 